Pacific Journal of Mathematics

SEPARATING CERTAIN PLANE-LIKE SPACES BY PLANO 


\title{
SEPARATING CERTAIN PLANE-LIKE SPACES BY PEANO CONTINUA
}

\author{
JoHN WM. GreEN
}

\begin{abstract}
Suppose $S$ is a connected, locally connected, complete Moore space having no cut point and in which the Jordan Curve Theorem holds. Thus, suppose $S$ satisfies R. L. Moore's Axioms 0, 1-4. Certain extensions and applications of earlier results of the author are established. In particular, modified forms of the Torhorst theorem and a plane theorem of $R$. $L$. Moore are shown to hold in the space $S$, two theorems concerning the separation of $S$ by compact dendrons are extended to Peano continua and another to certain Menger regular curves. Finally, a general method of constructing certain pathological spaces is given.
\end{abstract}

In [1], Gref showed that if the space $S$ is metrizable (which does not follow from the axioms indicated), which implies separability [5], [7], and no arc separates $S$, then many of the known separation theorems of plane topology can be established for the space $S$. His method of argument, however, is not valid in the present setting and indeed, many of the theorems he establishes are not true for the space here considered.

Extensive use is made of the results and terminology of [6] and [2]. The term "continuous curve" is to mean any connected, locally connected, closed point set, whether compact or not. A point $E$ of a continuous curve $M$ is an endpoint of $M$ if and only if $E$ is an endpoint of every arc in $M$ containing $E$. If $M$ is compact, this is equivalent to the definition in $[8, \mathrm{p} .64]$.

Theorem 1 (Modified Torhorst Theorem). If $U$ is a complementary domain of a Peano continuum $M$, there exists a Menger regular curve in $M$ which is an irreducible continuum about the boundary of $U$.

Proof. Let $\omega$ denote a point of $U$ and for each simple closed curve $J$ in $M$, let $D_{J}$ denote the interior of $J$ with respect to $\omega$; that is, let $D_{J}$ denote the complementary domain of $J$ not containing $\omega$. If $M$ contains no simple closed curve, the stated conclusion follows immediately from the fact that $M$ is a Menger regular curve and thus is hereditarily locally connected [8, p. 99]. Let $M^{\prime}$ denote the set of all points of $M$ not belonging to $D_{J}$ for any simple closed curve $J$ in $M$. Clearly, $M^{\prime}$ is a nonempty closed subset of the compact point set $M$. Suppose $D$ is a proper domain with respect to $M$ 
containing $M^{\prime}$. For each point $X$ of $M-D$, there is a simple closed curve $J$ in $M$ such that $D_{J}$ contains $X$. Since $M-D$ is compact and $D_{J}$ is open, there exists a finite collection $K$ of simple closed curves in $M$ such that every point of $M-D$ belongs to $D_{J}$ for some simple closed curve $J$ in $K$. It follows with the aid of Theorem 17, Chapter 3 of [6], that there exists a finite disjoint collection $L$ of simple domains covering $M-D$ such that each of them is $D_{J}$ for some simple closed curve $J$ in $M$. If $D_{J}$ belongs to $L$, it follows with the aid of Theorem 50, Chapter 1 of [6] that $M-D_{J}$ is connected, and a straightforward argument shows that $M-D_{J}$ is a continuous curve. It follows that $M-L^{*}$ is connected. $M-L^{*}$ is thus a connected, closed point set containing $M^{\prime}$ and lying in $D$. It follows that $M^{\prime}$ is connected. A modification of Jones' argument for Theorem 1 of [3] shows $M^{\prime}$ is locally connected.

Suppose $O$ and $P$ are points of $M^{\prime}$ which are not separated from each other in $M^{\prime}$ by any point. By Theorem 59, Chapter 2 of [6], there is a simple closed curve $J$ in $M$ containing $O$ and $P$. Let $E$ and $F$ denote points of $J$ such that $E+F$ separates $O$ from $P$ in $J$. If $E+F$ does not separate $O$ from $P$ in $M^{\prime}$, then $M^{\prime}$ contains a $\theta$ curve and this leads to a contradiction. Hence, $M^{\prime}$ is a regular curve. Let $N$ denote a subset of $M^{\prime}$ which is an irreducible continuum about the boundary of $U . N$ is a regular curve and each two points of $N$ are separated from each other in $N$ by some pair of points.

Theorem 2 (Modified Moore Theorem). If a Peano continuum $M$ separates the point $A$ from the point $B$, then $M$ contains an arc or a simple closed curve which separates $A$ from $B$.

Proof. By Theorem 1, there is a regular curve $N$ lying in $M$ which is an irreducible continuum about the boundary of the component $U$ of $S-M$ containing $A$. Let $V$ denote the component of $S-N$ containing $B$ and $N^{\prime}$ a subcontinuum of $N$ irreducible about the boundary of $V$. If the regular curve $N^{\prime}$ contains no simple closed curve, it follows from Theorem 5 of [2] that $N^{\prime}$ contains an arc which separates $A$ from $B$. If $N^{\prime}$ contains a simple closed curve $J$, it follows from Theorem 5 of [3] that $J$ separates $A$ from $B$.

It might at first appear that a similar argument would establish the stronger result that if $U$ is a complementary domain of a Peano continuum $M$ and $A$ is a point of $S-M-U$, then the outer boundary of $U$ with respect to $A$ is an arc or a simple closed curve. However, this is not true. The set $V$ of the argument above need not be a component of $S-\bar{U}$.

The next two theorems are sharper results than Theorems 9 and 
10 of [2].

THeOREM 3. If $U$ is a connected domain and $M$ is a Peano continuum which is an irreducible continuum about the boundary of $U$ and does not intersect $U$, then every endpoint of $M$ and every simple closed curve in $M$ is a subset of the boundary of $U$.

Proof. It follows from Theorem 1 that $M$ is a regular curve. Suppose $E$ is an endpoint of $M$ not on the boundary of $U$. There is a region $R$ containing $E$ but no boundary point of $U$. There is a point $A$ which separates $E$ from $M-R \cdot M$ in $M$. Let $H$ and $K$ denote mutually separated point sets whose sum is $M-A$ such that $H$ contains $M-$ $R \cdot M . H+A$ is a proper subcontinuum of $M$ containing the boundary of $U$. This is a contradiction. Suppose $J$ is a simple closed curve in $M$ which is not a subset of $\beta$, the boundary of $U$, and $D$ is the component of $S-J$ containing $U$. There is an arc $A X B$ on $J$ which contains no point of $\beta$. If there exists a free segment $T$ of $M$ lying in $J$, then $M-T$ is a proper subcontinuum of $M$ containing $\beta$. Thus, the branch points of $M$ are dense in $A X B$. Let $Y_{1}, Y_{2}$, $Y_{3}, \cdots$ denote branch points of $M$ in $A X B$ and $Y_{1} Z_{1}, Y_{2} Z_{2}, Y_{3} Z_{3}, \cdots \operatorname{arcs}$ in $M$ such that for each $n, Z_{n}$ is a boundary point of $U$ and $s\left(Y_{n} Z_{n}\right)$ is a subset of $D$. Suppose for some two integers $i$ and $j, Y_{i} Z_{i}$ and $Y_{j} Z_{j}$ have a point in common. Then $Y_{i} Z_{i}+Y_{j} Z_{j}$ contains an arc $Y_{i} O Y_{j}$ lying except for its endpoints in $D$. A contradiction results in much the same manner as in the argument for Theorem 2. Thus, if $i$ and $j$ are distinct integers, $Y_{i} Z_{i}$ and $Y_{j} Z_{j}$ are mutually exclusively arcs having one endpoint in $\beta$ and one endpoint in $A X B$. This contradicts Theorem 79, Chapter 2 of [6].

THEOREM 4. No endpoint of $S$ is a boundary point of three complementary domains of a Peano continuum.

Proof. Suppose the endpoint $E$ of $S$ is a boundary point of the three complementary domains $U_{1}, U_{2}$ and $U_{3}$ of the Peano continuum $M$. It follows from Theorem 2 above and Theorems 10, 12 and 13 of [2], that there is an arc $A_{1} E$ in $M$ which is irreducible with respect to being an arc which separates $U_{1}$ from $U_{2}$ and such that (1) $S-A_{1} E$ has only two components, one, $V_{1}$, containing $U_{1}$ and the other, $V_{2}$, containing $U_{2}$, (2) $A_{1} E$ is the boundary of $V_{1}$ and of $V_{2}$. One of the sets $V_{1}$ and $V_{2}$ contains $U_{3}$. Suppose that one is $V_{2} \cdot M \cdot\left(V_{2}+\right.$ $\left.E A_{1}\right)$ contains the boundaries of $U_{2}$ and $U_{3}$ and hence contains an arc $E A_{2}$ which separates $U_{2}$ from $U_{3}$ and which is a subset of the boundary of each of its complementary domains. Let $H_{2}$ and $H_{3}$ denote 
the components of $S-E A_{2}$ containing $U_{2}$ and $U_{3}$, respectively. One of the sets $H_{2}$ and $H_{3}$ contains $V_{1}$. Suppose that one is $\mathrm{H}_{3}$. Then $K_{2}=H_{2}$ is a component of $S-\left(E A_{1}+E A_{2}\right)$ and has boundary $E A_{2}$. $K_{1}=V_{1}$ is a component of $S-\left(E A_{1}+E A_{2}\right)$ with boundary $E A_{1}$. Let $K_{3}$ denote the component of $S-\left(E A_{1}+E A_{2}\right)$ containing $U_{3}$. There is an integer $i$, either 1 or 2 , such that $E A_{i}$ contains a sequence $X_{1}$, $X_{2}, X_{3}, \cdots$ of points converging to $E$ and such that for each $n, X_{n}$ is accessible from $K_{3}$. The set of all points of $E A_{i}$ accessible from $K_{i}$ is dense in $E A_{i}$. So there is a sequence $Y_{1}, Y_{2}, Y_{3} \ldots$ of points of $E A_{i}$ converging to $E$ and accessible from $K_{i}$. A slight modification of Jones' argument for Proposition 4 of [4] shows that a contradiction results.

THeorem 5. If $M$ is a Peano continuum and $U$ is a component of $S-M$ such that $B d(\bar{U})$ contains no endpoint of $S$, then $B d(U)$ is a Peano continuum.

Proof. There exists a regular curve $N$ in $M$ which is irreducible about the boundary of $\bar{U}$. It suffices to show that $N$ is a subset of the boundary of $U$. Suppose $X$ is a point of $N$. If $X$ is a boundary point of some component $V$ of $S-(N+\bar{U})$, then, since the boundary of $V$ is a simple closed curve $J$ by Theorems 2 and 3 above and Theorem 5 of [2], $X$ belongs to $J$ and $J$ lies in the boundary of $U$. Suppose $X$ is not a boundary point of any component of $S-(N+$ $\bar{U})$. By an argument similar to that for Theorem 8 of [2] using Theorem 25 of [4], it follows that no region is a subset of a compact regular curve. Thus, there exist components $D_{1}, D_{2}, D_{3}, \cdots$ of $S$ $(N+\bar{U})$ such that $X$ is a limit point of the sum of their boundaries $J_{1}, J_{2}, J_{3}, \cdots$, respectively. For each $n, J_{n}$ is a simple closed curve and hence is a subset of the boundary of $U$. Therefore, $X$ is a boundary point of $U$.

CoRollary. Under the hypothesis of Theorem 5, if $E$ is a component of $S-M$ other than $U$, then the outer boundary of $U$ with respect to $E$ is a simple closed curve.

Thus, Theorem 43, Chapter 4 of [6] remains true if Axiom 5 is replaced by the requirement that $M$ contain no endpoint of $S$.

THEOREM 6. If $U$ and $V$ are complementary domains of the continuous curve $M, E$ and $F$ are points of $M$ which are boundary points of both $U$ and $V$ and some arc from $E$ to $F$ in $M$ separates $U$ from $V$, then every arc from $E$ to $F$ in $M$ separates $U$ from $V$. 
Proof. Suppose $A$ and $B$ are two ares from $E$ to $F$ in $M$ such that $A$ separates $U$ from $V$ but $B$ does not. It follows from Theorems 10, 12 and 13 of [2] that $S-A$ has only two components and $A$ is the boundary of each of them. Let $U^{\prime}$ and $V^{\prime}$ denote these two components containing $U$ and $V$, respectively. There is an arc $C D$ not intersecting $B$ such that $C$ is in $U$ and $D$ is in $V$. Let $G$ denote the collection of all components of $A-A \cdot B$ which intersect $C D . G$ is finite since it is a disjoint collection of domains with respect to $A$ covering the compact closed set $A \cdot(C D)$. It follows from a slight modification of the argument for Theorem 110, Chapter 1 of [6] that there do not exist two elements $g_{1}$ and $g_{2}$ of $G$ and infinitely many components $t_{1}, t_{2}, t_{3}, \cdots$ of $C D-(C D) \cdot A$ such that for each $n, t_{n}$ has one endpoint in $g_{1}$ and one in $g_{2}$. Hence, there exist two finite sequences $s\left(L_{1} X_{1} R_{1}\right), s\left(L_{2} X_{2} R_{2}\right), \cdots, s\left(L_{n} X_{n} R_{n}\right)$ and $Z_{1}, Z_{2} \cdots, Z_{n}$ such that (1) for each $i \leqq n, \mathrm{~s}\left(L_{i} X_{i} R_{i}\right)$ is an element of $G$ containing the point $Z_{i}$, (2) the interval $X_{i} Z_{i}$ of $C D$ does not interset any element of $G$ distinct from $s\left(L_{i} X_{i} R_{i}\right)$, (3) $C X_{1}-X_{1}$ is a subset of $U^{\prime}$ and for each $i<n, \mathrm{~s}\left(Z_{i} X_{i+1}\right)$ and $Z_{n} D-Z_{n}$ do not intersect $A$, (4) $L_{i} X_{i} R_{i}$ is not $L_{i+1} X_{i+1} S_{i+1}$. (Other repetitions are possible.) For each $i \leqslant n$, let $s\left(L_{i} Y_{i} R_{i}\right)$ denote the component of $B-$ $A \cdot B$ with the endpoints indicated, let $J_{i}$ denote the simple closed curve $L_{i} Y_{i} R_{i} X_{i} L_{i}$ and $I_{i}$ the component of $S-J_{i}$ not containing $E$. $I_{i}$ intersects neither $U$ not $V$ and does not contain $F$.

$I_{1}$ is a subset of either $U^{\prime}$ or $V^{\prime}$. Suppose $I_{1}$ is a subset of $U^{\prime}$. There is an arc $R H T$ lying except for $R$ and $T$ in $V^{\prime}$ such that (1) the order $R X_{1} T$ is true on $s\left(L_{1} X_{1} R_{1}\right)$ and (2) the segment $R T$ of $A$ contains $(C D) \cdot\left(R_{1} X_{1} L_{1}\right)$. Let $I^{\prime}$ denote the complementary domain of the simple closed curve $J^{\prime}=L_{1} X_{1} R_{1} H L_{1}$ not containing $E . \quad I^{\prime}$ is a subset of $V^{\prime}$. Let $J^{\prime \prime}=R H T+L_{1} Y_{1} R_{1}+\left(L_{1} R_{1}-R T\right)$ and $I^{\prime \prime}$ its complementary domain not containing $E . I^{\prime \prime}=I_{1}+I^{\prime}+s(R T)$. Thus, $I^{\prime \prime}$ contains $X_{1} . C X_{1}$ does not intersect $L_{1} Y_{1} R_{1}$ since $C D$ does not intersect $B . C X_{1}$ does not intersect $L_{1} X_{1} R_{1}-R T$ since $R T$ contains $(C D) \cdot\left(L_{1} X_{1} R_{1}\right)$ and $C X_{1}$ does not intersect $s(R H T)$ since $s(R H T)$ lies in $V^{\prime}$ and $C X_{1}-X_{1}$ lies in $U^{\prime}$. Since $C X_{1}$ does not intersect $J^{\prime \prime}$, it is a subset of $I^{\prime \prime}$. It follows that $C$ does not belong to $U$. This is a contradiction. Therefore, $I_{1}$ is a subset of $V^{\prime}$.

There exists an arc $P O Q$ lying except for its endpoints in $U^{\prime}$ such that $s(P Q)$ of $s\left(L_{1} X_{1} R_{1}\right)$ contains $C D \cdot L_{1} X_{1} R_{1}$. Let $I^{\prime}$ denote the complementary domain of $P O Q X_{1} P$ not containing $E$. $I^{\prime}$ is a subset of $U^{\prime}$ and $I_{1}+I^{\prime}+s\left(P X_{1} Q\right)$ is the interior $I^{\prime \prime}$ with respect to $E$ of $J^{\prime \prime}=P O Q+L_{1} Y_{1} R_{1}+\left(L_{1} X_{1} R_{1}-P Q\right)$. The arc $Z_{1} X_{2}$ contains the point $Z_{1}$ of $I^{\prime \prime}$ and the point $X_{2}$ of $S-\bar{I}^{\prime \prime}$. Hence, $s\left(Z_{1} X_{2}\right)$ contains a point of $J^{\prime \prime}$. If $C^{\prime}$ is such a point, then $C^{\prime}$ is on $s(P O Q)$ and thus is in $U^{\prime}$. 
It follows that $s\left(Z_{1} X_{2}\right)$ is a subset of $U^{\prime}$. An induction argument using a modification of the argument above shows that $C D$ is a subset of $U^{\prime}+E F+I_{1}+I_{2}+\cdots+I_{n}$, so that $D$ is not a point of $V$. This is $a$ contradiction.

Theorem 7. If the Peano continuum $M$ separates $S$ and contains one of the complementary domains of each simple closed curve it contains, then every component of $S-M$ has an endpoint of $S$ on its boundary.

Proof. Suppose $U$ and $V$ are components of $S-M$ and $N$ is a regular curve which is an irreducible continuum about the boundary of $U$. By Theorem 2, $N$ contains an arc or simple closed curve which separates $U$ from $V$. Obviously, no simple closed curve in $N$ has this property. It follows from Theorem 5 of [2] that $N$ contains an endpoint of $S$, which by Theorem 3 is a boundary point of $U$.

The next result strengthens Theorem 21 of [2]. If the stipulation that $M$ be a compact regular curve is replaced by the stipulation that $M$ be a Peano continuum, the resulting conjecture is false.

THeorem 8. If the compact regular curve $M$ does not have infinitely many complementary domains but separates $S$, then there exist two components of $S-M$ with connected boundaries such that if $U$ is one of them, the boundary of $\bar{U}$ is a simple closed curve or a subset of an arc.

Proof. The argument procedes by induction on $n$, the number of components of $S-M$. The case $n=2$ follows easily with the aid of Theorems 12 and 13 of [2] and Theorem 2 above. Suppose the theorem is true for all integers $n$ greater than 1 and less than $k$ but is not true for $n=k$. Let the statement that the complementary domain $U$ of the closed point set $N$ has property $P$ relative to $N$ mean the boundary of $U$ is connected and the boundary of $\bar{U}$ is a simple closed curve or a subset of an arc. Let $M$ denote a compact regular curve such that $S-M$ has only $k$ components but does not have 2 components having property $P$ relative to $M$. If some component of $S-M$ has property $P$ relative to $M$, let $U$ denote it and if no such component exists, let $U$ denote any component of $S-M$.

Suppose there is an endpoint $E$ of $S$ on the boundary of $\bar{U}$. There is a component $V$ of $S-M-U$ whose boundary contains $E$ and there is a subcontinuum $N$ of $M$ not containing $E$ but containing the boundary of every component of $S-M$ other than $U$ and $V$. Since no compact regular curve contains a region, $S-N$ has only 
$k-1$ components and has no more than one component having property $P$ relative to $N$. This is a contradiction.

Suppose there is no endpoint of $S$ on the boundary of $\bar{U}$. It follows with the aid of Theorems 1 and 2 that there exists a subcontinuum $N$ of $M$ which is irreducible with respect to being a continuum which separates each two components $S-M$ from each other and which is the sum of a finite number of ares and does not contain infinitely many simple closed curves. It follows from Theorem 5 of [2] and Theorems 1 and 3 above that $N$ contains a simple closed curve $J$ which lies in the boundary of $U^{\prime}$, the component of $S-N$ containing $U . J$ contains a free segment $T$ of $N$ which does not intersect the boundaries of three components of $S-N . T$ does intersect the boundaries of two components of $S-N$, since otherwise, $N$ is not irreducible with respect to being a continuum which separates each two components of $S-M$ from each other. Let $N^{\prime}=N-T$. $N^{\prime}$ is a compact regular curve such that $S-N^{\prime}$ has only $k-1$ components but has no more than one component having proparty $P$ relative to $N^{\prime}$. This is a contradiction.

THEOREM 9. If $k$ and $n$ are nonnegative integers and $M$ is a Peano continuum which contains only $n$ simple closed curves and only $k$ endpoints of $S$, then $S-M$ has at most $n+k+1$ components.

Proof. Suppose $J$ is the only simply closed curve in $M$. Let $D$ and $I$ denote the components of $S-J$. The boundary of every component of $S-M$ contains either $J$ or an endpoint of $S$. It follows with the aid of Theorem 4 above and Theorem 4 of [3] that $S-M$ does not have infinitely many components. There is a subset $M^{\prime}$ of $M$ which is irreducible with respect to being a continuum which separates each two components of $S-M$ from each other. Neither $C l\left(C \cdot M^{\prime}\right)$ nor $C l\left(I \cdot M^{\prime}\right)$ contains $J$. Thus, each of these closed sets is a subset of a dendron in $M^{\prime}$. Let $k(D)$ and $k(I)$ denote the number of endpoints of $S$ in $D \cdot M^{\prime}$ and $I \cdot M^{\prime}$, respectively. It follows from a modification of Theorem 14 of [2] similar to Theorem 6 of [2] that $D-D \cdot M^{\prime}$ and $I-I \cdot M^{\prime}$ have no more than $k(D)+1$ and $k(I)+1$ components, respectively. Thus, $S-M^{\prime}$ has at most $k(D)+k(I)+2=k+2$ components. Hence, the theorem is true for $n=1$.

Suppose the theorem is true for $n<p$ and $M$ is a Peano continuum satisfying the hypothesis of the theorem for $n=p$. Define $M^{\prime}$ as above and let $J$ denote some simple closed curve in $M^{\prime}$. (If $M^{\prime}$ contains no simple closed curve, Theorem 14 of [2] gives the desired result.) It follows that there exist two components $U$ and $V$ of $S-$ $M^{\prime}$ separated from each other by $J$ and a free segment $T$ of $M^{\prime}$ in 
$J$ which is a subset $B d(U) \cdot B d(V)$ and intersects the boundary of no other component of $S-M^{\prime}$. $\quad M^{\prime}-T$ is a Peano continuum containing no more than $p-1$ simple closed curves, only $k$ endpoints of $S$ and has no more than $p+k$ complementary domains. Thus, $S-M$ has no more than $p+k+1$ components.

The next two theorems are very useful in constructing examples of spaces satisfying Axioms 0,1-4 and having endpoints. The proof of the first theorem is similar to arguments given in [2] and will be omitted.

THEOREM 10. If the ray $R$ contains no endpoints of $S$ and does not separate $S$, then $S-R$, with the subspace topology, satisfies Axioms 0, 1-4.

THEOREM 11. Suppose $S$ is the set of all points of a space satisfying Axioms 0,1-4, $J$ is a simple closed curve with complementary domains $D$ and $I, A E B$ is an are on $J, P$ is a point of $J-A E B$ accessible from $I, E F$ is an are lying except for $E$ in $I$ and $A_{1} B_{1}$, $A_{2} B_{2} \cdots$ is a sequence of mutually exclusive ares in $I$ converging to $A E B$, containing no endpoint of $S$ and such that for each $n, E_{n}$ is the only point of $A_{n} B_{n}$ on $E F$ and the order $F E_{n} E_{n+1} E$ on $E F$ holds. Suppose $M=(J-P-E)+D+\sum_{n}\left(A_{n} B_{n}-E_{n}\right)$. Suppose finally, that $S-M$, with the subspace topology, is locally connected at $P, E$ and $E_{n}$ for each n. Then the subspace $S-M$ satisjies Axioms 0,1-4 and $E$ is an endpoint of $S-M$.

Proof. Without loss of generality, it may be assumed that $F$ is not an endpoint of $S$. Since $M$ is the sum of countably many closed sets, $S-M$ is an inner limiting set. By Theorem 168, Chapter 1 of [6], the subspace $S-M$ satisfies Axioms 0 and 1. Clearly, $S-M$ satisfies Axiom 2.

Suppose $S-\bar{M}$ is not connecied. Let $U^{\prime}$ and $V^{\prime}$ denote components of $S-\bar{M}$. $\quad U^{\prime}$ and $V^{\prime}$ contain components $U$ and $V$, respectively, of $S-(\bar{M}+E F)$. Each component of $S-\left(\bar{M}+E F^{\prime}\right)$ intersects $A_{n} B_{n}-E_{n}$ for infinitely many $n$. There exist positive integers $k<$ $i<j<m$ and points $X_{i}$ on $A_{i} B_{i}-E_{i}, X_{j}$ on $A_{j} B_{j}-E_{j}, Y_{j}, Y_{k}$ on $A_{k} B_{k}-E_{k}$ and $Y_{m}$ on $A_{m} B_{m}-E_{m}$ such that $X_{i}$ and $X_{j}$ are accessible from $U$ and $Y_{k}$ and $Y_{m}$ are accessible from $V$. Let $X_{i} O X_{j}$ and $Y_{k} Q Y_{m}$ denote ares lying in $U$ and $V$, respectively. Let $J_{U}$ denote the simple closed curve formed by $X_{i} O X_{j}$, the intervals or points $X_{i} E_{i}$ of $A_{i} B_{i}$ and $X_{j} E_{j}$ of $A_{j} B_{j}$ and the interval $E_{i} E_{j}$ of $E F$, and let $I_{U}$ denote the interior of $J_{U}$ with respect to $D$. Let $J_{V}$ denote the simple closed curve formed by $Y_{k} Q Y_{m}, Y_{k} E_{k}, Y_{m} E_{m}$ and $E_{k} E_{m}$, and 
let $I_{V}$ denote the interior of $J_{V}$ with respect to $D . C l\left(I_{U} \cdot M\right)+E F$ is a compact dendron containing no endpoint of $S$ and whose intersection with $J_{U}$ is connected. By Theorem 6 of [2], $I_{U}-I_{U} \cdot \bar{M}$ is connected. It follows that $I_{U}-\bar{M}$ is a subset of $U$ and hence does not intersect $V$. Similarly, $I_{V}-\bar{M} \cdot I_{V}$ is a subset of $V$ and does not intersect $U$. Let $J^{\prime}$ denote the simple closed curve $E_{j} E_{m} Y_{m} Q Y_{k} E_{k} E_{i} X_{i} O X_{j} E_{j}$ and $I^{\prime}$ its interior with respect to $D . I^{\prime}=I_{U}+I_{V}+s\left(E_{i} E_{j}\right)$. It follows that $I^{\prime}-\bar{M} \cdot I^{\prime}$ is a connected subset of $S-\bar{M}$ intersecting both $U^{\prime}$ and $V^{\prime}$. This is a contradiction. Thus, $S-\bar{M}$ is connected.

Since $S-\bar{M}$ is connected, $P$ is not a cut point of $S-M$. Suppose some point $X$ is a cut point of $S-M$. There exists an arc $X E^{\prime}$ lying except for $E^{\prime}$ in $S-M-E F$ such that $E^{\prime}$ is on $E F$ but is not $E_{n}$ for any $n . M+\left(X E^{\prime}-E^{\prime}\right)$ has all the properties of $M$ used in the above argument to show $S-\bar{M}$ is connected. Thus, $S-$ $C l\left(M+X E^{\prime}\right)$ is connected. Hence, $S-M$ has no cut point.

Every arc in $S-M$ containing $E$ contains $E_{n}$ for sufficiently large n. For suppose $E X Q$ is an arc in $S-M$. Let $Z Q^{\prime}$ denote an arc lying except for $Z$ and $Q^{\prime}$ in $S-M$ such that $Z$ is on $J-A E B$ and $Z Q^{\prime}$ is irreducible from $J$ to $E X Q$. Let $J_{A}$ denote the simple closed curve formed by the arc $Z Q^{\prime}$, the interval $Q^{\prime} E$ of $E X Q$ and the arc $Z A E$ on $J$ and let $I_{A}$ denote the interior of $J_{A}$ with respect to $D$. Let $W O^{\prime}$ denote an arc lying except for $W$ and $O^{\prime}$ in $S-\bar{M}$ such that $W$ is on $J-Z E B$ and $W O^{\prime}$ is irreducible from $J$ to $J_{A}$. Let $J_{B}$ denote the simple closed curve formed by the arc $W O^{\prime}$, the arc $O^{\prime} E$ of $J_{A}$ not containing $A$ and the arc $E B W$ on $J$ and let $I_{B}$ denote the interior of $J_{B}$ with respect to $D . I_{A}$ and $I_{B}$ are mutually exclusive. It follows that $I_{A}+D+s(Z A E)$ is a domain containing $A E-E$ and $I_{B}+D+s(W B E)$ is a domain containing $B E-E$. Since the sequence $A_{1} B_{1}, A_{2} B_{2}, \cdots$ converges to $A E B$ and does not intersect $D$, it follows that for sufficiently large $n, A_{n} B_{n}$ intersects both $I_{A}$ and $I_{B}$. Hence, for sufficiently large $n, A_{n} B_{n}$ intersects $E X Q$. Since $E X Q$ is a subset of $S-M$, it follows that $E_{n}$ is eventually on $E X Q$. It follows $E$ is an endpoint of $S-M$.

It remains to be shown that Axiom 4 is satisfied. Suppose $T$ is a simple closed curve in $S-M$ not containing $P$. There is a positive integer $N$ such that $T$ does not intersect $E E_{N}$. Let $D_{T}$ and $I_{T}$ denote the interior and exterior, respectively, of $T$ with respect to $E$. There is an arc $C$ on $T$ such that $H=C$ plus all components of $C l\left(I_{T} \cdot M\right)$ intersecting $T$ is a compact dendron. It follows from Theorem 6 of [2] that $I_{T}-H \cdot I_{T}$ is connected. $I_{T}$, as a subspace of $S$, satisfies Axioms 0,1-4 by Theorem 23, Chapter 3 of [6]. In this subspace, each component of $C l\left(I_{T} \cdot M\right)$ intersecting $T$ is a ray containing no endpoint of the subspace and, from the argument above, 
does not separate the subspace. Since there are not infinitely many of these rays, $I_{T}-I_{T} \cdot H$ satisfies Axioms $0,1-4$, by Theorem 10 . The first part of the proof of this theorem now applies to the subspace $I_{T}-I_{T} \cdot H$, with $M$ replaced by $M \cdot I_{T}-H \cdot I_{T}$, to show that $I_{T}-I_{T} \cdot M$ is connected. Clearly, $T$ is the boundary with respect to $S-M$ of $I_{T} \cdot \quad C l\left(M \cdot D_{T}\right)$ is the sum of a finite number of arcs. It follows that there is a compact dendron $M^{\prime}$ containing $C l\left(M \cdot D_{T}\right)$, lying in $\bar{D}_{T}$, intersecting $T$ in a connected set and containing no endpoint of $S$. By Theorem 6 of [2], $D_{T}-M^{\prime} \cdot D_{T}$ is connected. $T$ is the boundary with respect to $S-M$ of $D_{T}$. Thus, $S-M-T$ is the sum of two mutually exclusive connected domains each having boundary $T$.

Suppose $T$ is a simple closed curve in $S-M$ containing $P$. Define $I_{T}$ and $D_{T}$ as before. As above, $D_{T}-M \cdot D_{T}$ is connected and $T$ is the boundary with respect to $S-M$ of $D_{T}$ and $I_{T}$. Suppose $I_{T}-\bar{M} \cdot I_{T}$ is not connected and $U^{\prime}$ and $V^{\prime}$ are two of its components. For each $n, H_{n}=J+T+\left(E_{n} F+A_{1} A_{1}+A_{2} B_{2}+\cdots+A_{n} B_{n}\right) . \quad \bar{I}_{T}$ is a compact regular curve containing no endpoint of $S$ and only two simple closed curves. Hence, it has at most three complementary domains by Theorem 9. $D, I_{T}-\bar{D}-H_{n}$ and $D_{T}-H_{n}$ are three mutually separated sets whose sum is $S-H_{n}$. Thus, these three sets are connected. In particular, $I_{T}-\bar{D}-H_{n}$ is connected. It follows that the boundaries of $U^{\prime}$ and $V^{\prime}$ intersect $A_{n} B_{n}-E_{n}$ for infinitely many $n$. A modified form of the argument in the second paragraph of this proof shows that a contradiction results. This completes the proof.

\section{REFRENCES}

1. Lynn George Gref, Consequences of the Jordan Curve Theorem, Unpublished University of California, Riverside, Disertation, 1966.

2. J. Wm. Green, Concerning the separation of certain planelike spaces by compact dendrons, Duke Math. J., 38 (1970), 555-571.

3. F. B. Jones, Concerning the boundary of a complementary domain of a continuous curve, Bull. Amer. Math. Soc., 45 (1939), 428-435.

4. - Certain Consequences of the Jordan Curve Theorem, Amer. J. Math., 63 (1941), 531-544.

5. - Concerning the separability of certain locally connected metric spaces, Bull. Amer. Math. Soc., 52 (1946), 303-306.

6. R. L. Moore, Foundations of Point Set Theory, Amer. Math. Soc. Colloquium Pub., XIII, revised edition, 1962.

7. Prabir Roy, Separability of connected, locally connected metric spaces, Duke Math.

J., 29 (1962), 99-100.

8. G. T. Whyburn, Analytic Topology, Amer. Math. Soc. Colloquium Pub., XXVIII, 1946.

Received September 14, 1970.

UNIVERSITY OF OKLAHOMA 


\title{
PACIFIC JOURNAL OF MATHEMATICS
}

\author{
EDITORS
}

\author{
H. Samelson \\ Stanford University \\ Stanford, California 94305 \\ C. R. HOBBY \\ University of Washington \\ Seattle, Washington 98105
}

J. DugundJI

Department of Mathematics

University of Southern California

Los Angeles, California 90007

RICHARD ARENS

University of California

Los Angeles, California 90024

\section{ASSOCIATE EDITORS}

E. F. BECKENBACH
B. H. NEUMANN

F. WoLF
K. YosHIDA

\section{SUPPORTING INSTITUTIONS}

\author{
UNIVERSITY OF BRITISH COLUMBIA \\ CALIFORNIA INSTITUTE OF TECHNOLOGY \\ UNIVERSITY OF CALIFORNIA \\ MONTANA STATE UNIVERSITY \\ UNIVERSITY OF NEVADA \\ NEW MEXICO STATE UNIVERSITY \\ OREGON STATE UNIVERSITY \\ UNIVERSITY OF OREGON \\ OSAKA UNIVERSITY \\ UNIVERSITY OF SOUTHERN CALIFORNIA
}

\author{
STANFORD UNIVERSITY \\ UNIVERSITY OF TOKYO \\ UNIVERSITY OF UTAH \\ WASHINGTON STATE UNIVERSITY \\ UNIVERSITY OF WASHINGTON \\ $\stackrel{*}{*} \stackrel{*}{*} \stackrel{*}{*}{ }^{*}{ }^{*}$ MERICAN MATHEMATICAL SOCIETY \\ CHEVRON RESEARCH CORPORATION \\ NAVAL WEAPONS CENTER
}

The Supporting Institutions listed above contribute to the cost of publication of this Journal, but they are not owners or publishers and have no responsibility for its content or policies.

Mathematical papers intended for publication in the Pacific Journal of Mathematics should be in typed form or offset-reproduced, (not dittoed), double spaced with large margins. Underline Greek letters in red, German in green, and script in blue. The first paragraph or two must be capable of being used separately as a synopsis of the entire paper. The editorial "we" must not be used in the synopsis, and items of the bibliography should not be cited there unless absolutely necessary, in which case they must be identified by author and Journal, rather than by item number. Manuscripts, in duplicate if possible, may be sent to any one of the four editors. Please classify according to the scheme of Math. Rev. Index to Vol. 39. All other communications to the editors should be addressed to the managing editor, Richard Arens, University of California, Los Angeles, California, 90024.

50 reprints are provided free for each article; additional copies may be obtained at cost in multiples of 50 .

The Pacific Journal of Mathematics is published monthly. Effective with Volume 16 the price per volume (3 numbers) is $\$ 8.00$; single issues, $\$ 3.00$. Special price for current issues to individual faculty members of supporting institutions and to individual members of the American Mathematical Society: $\$ 4.00$ per volume; single issues $\$ 1.50$. Back numbers are available.

Subscriptions, orders for back numbers, and changes of address should be sent to Pacific Journal of Mathematics, 103 Highland Boulevard, Berkeley, California, 94708.

PUBLISHED BY PACIFIC JOURNAL OF MATHEMATICS, A NON-PROFIT CORPORATION

Printed at Kokusai Bunken Insatsusha (International Academic Printing Co., Ltd.), 7-17, Fujimi 2-chome, Chiyoda-ku, Tokyo, Japan. 


\section{Pacific Journal of Mathematics}

J. T. Borrego, Haskell Cohen and Esmond Ernest Devun, Uniquely

representable semigroups on the two-cell .................. 565

Glen Eugene Bredon, Some examples for the fixed point property ........ 571

William Lee Bynum, Characterizations of uniform convexity .......... 577

Douglas Derry, The convex hulls of the vertices of a polygon of order $n \ldots 583$

Edwin Duda and Jack Warren Smith, Reflexive open mappings .......... 597

Y. K. Feng and M. V. Subba Rao, On the density of $(k, r)$ integers ........ 613

Irving Leonard Glicksberg and Ingemar Wik, Multipliers of quotients of

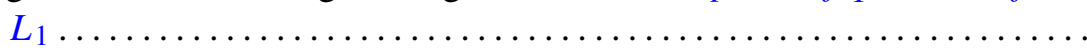

John William Green, Separating certain plane-like spaces by Peano

continua.........................................

Lawrence Albert Harris, A continuous form of Schwarz's lemma in normed

linear spaces .................................... 635

Richard Earl Hodel, Moore spaces and $w$-spaces ............... 641

Lawrence Stanislaus Husch, Jr., Homotopy groups of PL-embedding spaces.

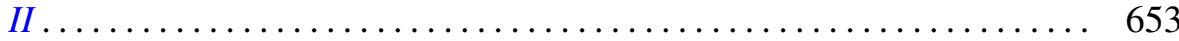

Yoshinori Isomichi, New concepts in the theory of topological

space-supercondensed set, subcondensed set, and condensed set.....

J. E. Kerlin, On algebra actions on a group algebra .................

669

Keizō Kikuchi, Canonical domains and their geometry in $C^{n} \ldots \ldots \ldots \ldots 681$

Ralph David McWilliams, On iterated $w^{*}$-sequential closure of cones. .

697

C. Robert Miers, Lie homomorphisms of operator algebras ..

717

Louise Elizabeth Moser, Elementary surgery along a torus knot ...

737

Hiroshi Onose, Oscillatory properties of solutions of even order differential

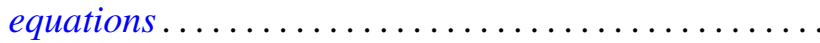

747

Wellington Ham Ow, Wiener's compactification and $\Phi$-bounded harmonic

functions in the classification of harmonic spaces...

Zalman Rubinstein, On the multivalence of a class of meromorphic

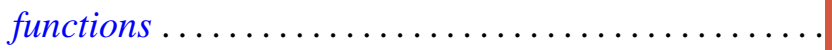

771

785

Hans H. Storrer, Rational extensions of modules

795

topological lattices ...........................

Robert Evert Stong, On the cobordism of pairs .................. 803
Albert Leon Whiteman, An infinite family of skew Hadamard matrices . . . 817

Lynn Roy Williams, Generalized Hausdorff-Young inequalities and mixed

norm spaces 